\title{
Gelungene Digitalisierung braucht funktionierende Schnittstellen
}

\author{
Alexander Zimmer \\ Dr. med., Mitglied des Zentralvorstandes und Departementsverantwortlicher Digitalisierung/eHealth
}

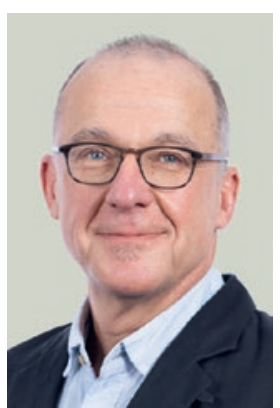

Haben Sie es auch schon bemerkt? Hersteller von Smartphones, Tablets oder Monitoren setzen zunehmend auf randlose Displays. Dies zum Ärger derjenigen, die es gewohnt sind, Notizzettel auf den Monitor ihres Gerätes zu heften. Einzige Ausnahme ist der Hersteller mit dem Apfel, dessen Bildschirm am unteren Rand eine Fläche besitzt, an der sich weiterhin die Klebezettel mit den handschriftlichen Daten anbringen lassen.

Die digitale Transformation wird von ihren Akteuren unterschiedlich umgesetzt. Das zeigt sich auch auf der Ebene der Bundesbehörden.

Die anfänglichen Schwierigkeiten in der Bewältigung der Covid-Pandemie haben zu einigen parlamentarischen Vorstössen geführt, die sich für eine Stärkung der Digitalisierung im Gesundheitswesen aussprechen. Der Nachholbedarf ist hoch, auch angesichts der Tatsache, dass die Schweiz im Digital-Health-IndexRanking der Bertelsmann-Stiftung abgeschlagen auf Rang 14 von 17 Ländern verharrt. Das Bundesamt für Gesundheit hat prompt reagiert und ihre eHealthStrategie mit der Gründung einer Abteilung Digitale Transformation bekräftigt. Noch in der sechsten Nationalen Konferenz «Gesundheit2020» im Jahr 2019 wurde festgehalten, dass die Digitalisierung die Grundlagen für ein besseres, sichereres und effizienteres Gesundheitswesen schaffen und insbesondere Gesundheitsfachpersonen und Institutionen vernetzen soll.

Für eine gelungene digitale Transformation ohne massiven administrativen Mehraufwand brauchen wir funktionierende Schnittstellen.

So weit, so gut. Auf der Website des BAG für meldepflichtige Infektionskrankheiten findet sich aber auch im zweiten Jahr der aktuellen Pandemie der Hinweis darauf, dass Laboratorien epidemiologische Befunde mittels Meldeformular per Fax oder auf dem Postweg an das BAG einsenden sollen. Immerhin wird auch darauf verwiesen, dass das BAG eine elektronische Labormeldung einführt.

Dabei wäre doch der Mehrwert der Digitalisierung gerade, dass Daten, die einmal erfasst wurden, für weitere Zwecke wiederverwendet werden können. Dies erspart uns Ärztinnen und Ärzten eine doppelte Buchführung, die mit der zusätzlichen Erfassung von digitalen Formularen entsteht.

Die FMH hat sich im Zusammenhang mit der Beteiligung an der künftigen AD-Swiss-EPD-Gemeinschaft dafür engagiert, dass Ärztinnen und Ärzte die in ihrem üblichen medizinischen Arbeitsalltag anfallenden und im eigenen Primärsystem erfassten Daten wiederverwenden können - beispielsweise für die Meldung von Impfdaten an das BAG. Und dies ohne eine erneute Eingabe der Daten in ein zusätzliches Meldeformular.

Leider wird diese Lösung nicht von allen Kantonen unterstützt, da sie teilweise auf ihren eigenen Lösun gen zur Erfassung der Impfdaten beharren. Ein Schelm, wer Böses dabei denkt. Sollte etwa die direkte Übermittlung von Daten aus dem Primärsystem von Ärztinnen und Ärzten an das BAG zur Erfüllung ihrer Meldepflicht dem Aufbau eigener Impfregister auf Verwaltungs- oder Kantonsebene entgegenstehen? Das Prinzip der einmaligen Datenerfassung und mehrfachen Nutzung bedingt nicht nur bessere Kontrollmöglichkeiten durch Patientinnen und Patienten. Die Datenautonomie der Ärzteschaft aufrechtzuerhalten und zu fördern ist im Zeitalter der zunehmenden Digitalisierung ein grosses Anliegen der FMH. Im Tarifbereich profitiert die Ärzteschaft bereits von den via Trustcentren und NewIndex gesammelten Daten.

Auf den ersten Blick haben die eingangs erwähnten Haftzettel durchaus Vorteile: Sie sind schnell einsetzbar und daher effizient nutzbar. Aber sie stehen auch sinnbildhaft dafür, dass die digitale Transformation noch nicht vollständig nutzenbringend umgesetzt wird. Spätestens dann, wenn die darauf befindlichen Informationen digital ausgetauscht oder wiederverwendet werden sollen, müssen sie, wie bei einer doppelten Buchführung, erneut eingegeben und dann übertragen werden. Das ist ein unnötiger Aufwand. Aus diesem Grund setzt sich die FMH mit Nachdruck für die Lösung der Schnittstellenproblematik und den damit verbundenen nutzenbringenden Austausch von strukturierten medizinischen Daten ein. 